\title{
Response of Mn overlayers on Fe to external magnetic fields: Electronic structure calculations
}

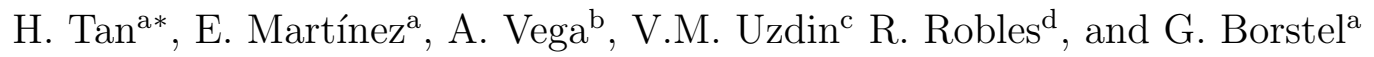 \\ ${ }^{a}$ Fachbereich Physik, Universität Osnabrück \\ D-49080 Osnabrück, Germany

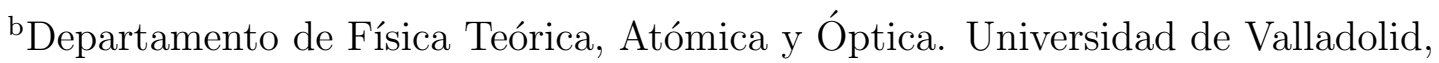 \\ E-47011 Valladolid, Spain
}

${ }^{\mathrm{c}}$ St. Petersburg State University, Universitetskaya nab. 7/9,

St. Petersburg, 199178 Russia

${ }^{\mathrm{d}}$ Department of Physics, Virginia Commonwealth University,

Richmond, Virginia 23284, USA

The behavior of $\mathrm{Mn}$ overlayers on $\mathrm{Fe}(001)$ under the influence of external magnetic fields is investigated. The electronic charge distribution, local magnetic moments as well as their couplings are determined as a function of the external field by solving self-consistently a tight binding Hamiltonian, parameterized to ab-initio TBLMTO calculations. Our method allows to trace back the field-dependent average magnetization of the system to its electronic structure and magnetic configuration. We show how in the noncollinear framework the response of the system is markedly different to what is found in the collinear framework. If metastable magnetic configurations exist, the external field can be used for tuning the system between some of them because the system stays in some of those metastable states even after switching off the external field.

Keywords: Semi-empirical models and model calculations; Haydock's recursion method; manganese layers on iron substrate; magnetic surface and interface

\section{Introduction}

The magnetism of a large variety of nanostructures has been investigated in the last decades from both experimental and theoretical sides [1-4]. The full un-

*Corresponding author. Tel.: +49-541-9692620; fax: +49-541-969-2351. E-mail address: htan@uos.de (H. Tan). derstanding of the magnetic properties of a given system requires not only knowing its intrinsic properties, but also its response to external fields. Moreover, many of the technological applications of magnetism are based on the possibility of engineering the system by the application of external fields [5]. Experimentally, the use of external magnetic fields is 
a common practice which helps to both purposes. The investigation of the response to external magnetic fields has been carried out through various experimental techniques, like magneto-optical Kerr effect [6], magnetic transmission Xray microscopy [7], Fresnel and Foucault microscopy [8], Lorentz microscopy [9] or ballistic electron magnetic microscopy [10]. All those techniques have limited resolution, reaching in some cases down to $40 \mathrm{~nm}$. To increase the resolution, the recently developed Spin-Polarized Scanning Tunneling Microscopy can be used, making possible the observation of hysteresis at the nanometer scale [11]. Increasing the level of locality of the applied fields is a challenge which will improve the density of information storage in recording media and, consequently, the device miniaturization.

From the theoretical side, most of the studies and simulations of nanomagnetism are associated with the investigation of the intrinsic properties of the system. The response of magnetic systems to external fields has been less investigated. Usually, this response is described on the basis of phenomenological models, due to the complexity of the nanostructures of interest. For example, magnetic fields have been used in theory as an instrument to select specific solutions (in particular those corresponding to the spin density waves) from a set of self-consistent ones [12]. In that study, a magnetic field was introduced in a collinear model so that all moments were ordered either along or opposite to the field. Such situation can be ex- perimentally realized in single molecular magnets [15]. These systems contain superparamagnetic 3d-clusters (for example $\mathrm{Mn}_{12}$ or $\mathrm{Fe}_{8}$ ), and their total magnetic moments show a strong uniaxial anisotropy. Molecular magnets demonstrate resonant quantum tunnelling between states with different spin projections having the same energy in external magnetic fields. This leads to step-like hysteresis loops at low temperatures. However, in general, a noncollinear theory is necessary for an adequate description of the system response to an external magnetic field. In systems where the magnetic anisotropy is smaller than in molecular magnets, the remagnetization process on an external field involves the rotation of magnetic moments, which can only be studied under a noncollinear approach. On the other hand, noncollinear magnetism has attracted considerable attention in recent years and has been found in many systems like exchange spring magnets [16], geometrically constrained domain walls [2], magnetic pendulums in thin wires [3,4], or supported clusters $[13,14]$. In nanostructures, noncollinear states can appear as a result of the combination of magnetic ordering and geometrical confinement. The response of these states to external magnetic fields may be very rich and its study can shed light on the behavior of nanostructures in real devices.

Antiferromagnetic structures on ferromagnetic substrates are interfacial systems that have attracted considerable attention[17,18]. One of the systems intensively investigated experimentally $[19,20]$ 
as well as theoretically [21-23] is Mn supported on Fe. The fact that Mn is located in the periodic table between $\mathrm{Fe}$ (ferromagnetic) and $\mathrm{Cr}$ (antiferromagnetic) gives rise to a large variety of local magnetic couplings in the collinear framework. For instance, in the experiments of Yamada et al.[19] and Schlickum et al.[20] only the $p(1 \times 1)$ configuration at the surface is found, but there are some theoretical studies in which antiferromagnetic $c(2 \times 2)$ configurations are proposed [22]. Concerning the magnetic coupling at the $\mathrm{Mn} / \mathrm{Fe}$ interface, some theoretical studies found this coupling to be ferromagnetic [21,22], while other studies proposed an antiferromagnetic coupling [23]. This makes this system particularly interesting as regards the possibility of noncollinear magnetism and its response to external magnetic fields. For instance, the presence of monoatomic steps at the substrate leads to noncollinear effects [24], with the development of surface magnetic domains and domain walls. The rich magnetic behavior of Mn films supported on Fe and the high spin-polarization of both elements make particularly interesting to study the response of the system to external magnetic fields, although the applied magnetic fields are expected to be very high, due to the difficulty to overcome the magnetic couplings between layers in the system. Furthermore, this system is an excellent choice in order to perform a comparative analysis of collinear and noncollinear pictures.

In this paper, we report a detailed study of the magnetic structure of six Mn layers supported on $\mathrm{Fe}(001)$ and its response to external magnetic fields. Our theoretical method is described in section 2. In the next sections we compare the results obtained in both collinear and noncollinear frameworks. We start discussing the intrinsic magnetic properties (in the absence of external fields) and then we analyze the response of the system to an uniform external magnetic field. The main conclusions are summarized at the end.

\section{Theoretical method}

Our semi-empirical theoretical model is based on the fully self-consistent Tight Binding (TB) method that was used in our previous studies of supported $\mathrm{Cr}$ and $\mathrm{Mn}$ films over Fe $[24,26,27]$. Here we have extended this method to account for the interaction of the spin-moments with an external magnetic field. A similar approach have been used by part of us to describe the remagnetization process in exchange spring magnets [25]. The method allows to consider both uniform external fields and non-uniform fields, i.e., fields acting with different intensity and orientation depending on the atomic site in the system. This last implementation would allow to perform simulations of future experiments in which local magnetic fields will be applied over selected parts of the system, even at the atomic scale, a situation that is not the aim of the present work, but that will be explored in future studies.

Our TB method is parameterized through a mapping to the $a b$ initio TBLMTO [28] Hamiltonian in the first order expansion of its Tight-Binding form 
and in the Slater-Koster approximation, from which the two-center hopping integrals can be extracted. The spin quantization axis is independent in each site, thus allowing to describe noncollinear magnetism. The electronic charge distribution and the local magnetic moments in the system are obtained by Haydock's recursion method [29] to self-consistently solve the resulting one-particle TB Hamiltonian for the $s, p$, and $d$ valence electrons in a mean-field approximation (equivalent to the unrestricted Hartree-Fock approximation) which retains the main electronic correlations effects to accurately account for the itinerant magnetism in transitionmetal systems at $T=0 K$.

The Hamiltonian can be split into three terms: a magnetism-independent term $H_{\text {ind }}$, an exchange term $H_{\text {mag }}$, and a term containing the interaction with the external magnetic field $H_{\text {Bext }}$. A localized orthogonal atomic orbital basis $\{|i \alpha\rangle\}$ is introduced to represent in matrix form the Hamiltonian $H=H_{\text {ind }}+H_{\text {mag }}+H_{\text {Bext }}$, where

$$
\begin{aligned}
H_{\text {ind }}= & \sum_{\substack{i, j \\
\alpha, \beta}}\left[\left(\epsilon_{i \alpha}^{0} \delta_{\alpha \beta}+U_{i \alpha, j \beta}\left\langle\hat{n}_{j \beta}\right\rangle\right.\right. \\
& \left.+Z_{i} \Omega_{i \alpha} \delta_{\alpha \beta}\right) \delta_{i j}+ \\
& \left.t_{i j}^{\alpha \beta}\left(1-\delta_{i j}\right)\right]|i \alpha\rangle\langle j \beta|\left[\begin{array}{cc}
1 & 0 \\
0 & 1
\end{array}\right] \\
H_{m a g}= & \sum_{i, \alpha, \beta}\left(-\frac{1}{2} J_{i \alpha \beta} \mu_{i \alpha}\right)|i \beta\rangle\langle i \beta| \\
& \times\left[\begin{array}{cc}
\cos \theta_{i} & e^{-i \phi_{i}} \sin \theta_{i} \\
e^{i \phi_{i}} \sin \theta_{i} & -\cos \theta_{i}
\end{array}\right]
\end{aligned}
$$

$$
\begin{aligned}
H_{\text {Bext }}= & -\sum_{i, \alpha} \frac{g_{s} \mu_{B}}{\hbar} \vec{B}_{i} \vec{S}_{i}|i \alpha\rangle\langle i \alpha| \\
= & -\frac{1}{2} g_{s} \mu_{B} \sum_{i, \alpha}|i \alpha\rangle\langle i \alpha| \\
& \times\left[\begin{array}{cc}
B_{i}^{z} & B_{i}^{x}-i B_{i}^{y} \\
B_{i}^{x}+i B_{i}^{y} & -B_{i}^{z}
\end{array}\right]
\end{aligned}
$$

$H_{\text {ind }}$ contains both diagonal and nondiagonal magnetism-independent matrix elements. The hopping integrals $t_{i j}^{\alpha \beta}$ between orbitals $\alpha$ and $\beta$ of different sites $i$ and $j$ are assumed, as usual, to be spin independent. These hopping integrals take implicitly into account, through their parameterization to the TB-LMTO Hamiltonian of supported Mn films on $\mathrm{Fe}(001)$, the effects of the reduced coordination at the surface and the $\mathrm{Mn}-\mathrm{Fe}$ hybridization at the interface. The magnetism-independent diagonal elements $\epsilon_{i \alpha}^{0}+U_{i \alpha, j \beta}\left\langle\hat{n}_{j \beta}\right\rangle+Z_{i} \Omega_{i \alpha}$, are the sum of: $i$ ) the orbital level of the isolated atom $\left.\epsilon_{i \alpha}^{0}, i i\right)$ the electrostatic level shift $U_{i \alpha, j \beta}\left\langle\hat{n}_{j \beta}\right\rangle$, accounting for the electronic charge redistribution which is parameterized by the Coulomb integral $U_{i \alpha, j \beta}$, and the spin-explicit form of the Coulomb integral is $U_{i \alpha \beta}=\sum_{i} U_{i \alpha \beta} \delta_{i j}=$ $\left(U_{i \alpha \uparrow, i \beta \uparrow}+U_{i \alpha \uparrow, i \beta \downarrow}\right) / 2$, and $\left.i i i\right)$ the crystal field potential which, as a first approximation, reads $Z_{i} \Omega_{i \alpha}$, where $Z_{i}$ is the local atomic coordination of site $i$ and $\Omega_{i \alpha}$ is a potential which depends on the orbital character (localized like the $d$ orbitals or delocalized like the $s p$ ones).

$H_{\text {mag }}$ describes the magnetic effects in a molecular field picture through the exchange parameter $J_{i \alpha}$ times the local mag- 
netic moment, $\mu_{i \alpha}$, whose direction is given by the angles $\left(\theta_{i}, \phi_{i}\right)$ defined in the spin-rotation matrix which is applied to the spin basis at each atomic site. Notice that, in the noncollinear framework, the spin-quantization axis is independent at each site. The spin-explicit form of the exchange parameter is defined as $J_{i \alpha \beta}=$ $\left(U_{i \alpha \uparrow, i \beta \downarrow}-U_{i \alpha \uparrow, i \beta \uparrow}\right)$.

$H_{B e x t}$ describes the interaction of the local spin magnetic moments $\overrightarrow{\mu_{i}}=g_{s} \mu_{B} \overrightarrow{S_{i}}$ with the external magnetic field $\vec{B}_{i}, g_{s}$ being the gyromagnetic factor and $\mu_{B}$ the Bohr magneton. In the present work we focus on the response to uniform external fields and, therefore, for the calculations it is pertinent to choose the direction of the external magnetic field (which is the same for all atomic sites) as the direction of the reference (laboratory) quantization axis $z$, to which the local spin-quantization axis are rotated. In such conditions, the matrix in equation (3) has a diagonal form, since $\sigma_{x}$ and $\sigma_{y}$ Pauli matrices do not enter in the expression.

The local densities of states (LDOS) are calculated through the retarded Green's function using the recursion method [29] in the real space which provides the local orbital projected densities of states (LDOS) on an arbitrary local quantization axis through a rotation of the initial recursion vector in the spin space. We calculate electronic occupations and magnetic moments at each orbital and site of the system by integrating the LDOS up to the Fermi level, with the global charge neutrality condition. All the parameters of the TB model as well as the interlayer distances have been taken as in Ref.[24]. The hopping integrals for surface manganese layer, intermediate manganese layer, interface manganese layer,interface iron layer and other iron layers are taken differently to distinguish the local environment of different atoms.

Self-consistency requires that the effective potential entering in the Hamiltonian leads to a charge and magnetic moment distribution which generates the same effective potential. We compute the electronic charges and magnetic moments from the retarded Green's function as described before, to be fed into the local effective potential leading to a new Green's functions from which the charge and moment distributions can be again calculated. When the electronic charges, local moments (the three components in the noncollinear framework) and the total energy differ from one iteration to the next in less than a given accuracy, the selfconsistency is reached.

It is worth analyzing the orders of magnitude involved in the interaction with the external magnetic field. $1 T=5.789 \times$ $10^{-5} \mathrm{eV} / \mu_{B}$. This means that extremely high external fields are needed to overcome typical exchange couplings in transition metals. This is expected to come out from our model, particularly in the collinear framework where there is no possibility for the local moments to rotate towards the external field. 


\section{Collinear results}

\subsection{Intrinsic magnetic properties}

We start with the analysis of the magnetic properties of six Mn layers supported on the Fe(001) substrate. If we consider a $p(1 \times 1)$ configuration, in which the magnetic moment of each Mn layer can point either in the $+z$ direction $(+)$ or in the $-z$ direction $(-)$, there are $2^{6}=64$ possible collinear magnetic configurations in the Mn slab. Some of them have been already studied in previous works by means of $a b$ initio DFT (Density Functional Theory) calculations $[21,22]$.

In Table 1 we report those collinear magnetic configurations which are solutions of the self-consistent calculation. Starting from the 64 possibilities, we have obtained 23 configurations (the other 41 inputs converge during the selfconsistency to some of the solutions listed in Table 1). Both parallel and antiparallel couplings between Mn layers as well as at the $\mathrm{Mn} / \mathrm{Fe}$ interface exist. The ground state in the collinear framework shows antiparallel couplings between Mn layers and a parallel coupling at the $\mathrm{Mn} / \mathrm{Fe}$ interface, in agreement with experimental results[19,20]. As a general trend, we find that those metastable configurations which are energetically close to the ground state have antiparallel coupling between the Mn-surface and subsurface layers as well as between the Mn layers adjacent to the Fe interface, and a parallel coupling at the $\mathrm{Mn} / \mathrm{Fe}$ interface. A variety of metastable configurations corresponding to different magnetic couplings in $\mathrm{Mn}$ (particularly in the central lay-

\begin{tabular}{cccc}
\hline \hline state & $\mathrm{E}(\mathrm{eV})$ & $\bar{\mu}\left(\mu_{B}\right)$ & configuration \\
\hline 0 & 0.000 & -0.16 & -+-+-+ \\
1 & 0.088 & 0.22 & +--+-+ \\
2 & 0.117 & 1.12 & +-++-+ \\
3 & 0.199 & 0.12 & +-+-+- \\
4 & 0.209 & 0.31 & +-+--+ \\
5 & 0.270 & -0.28 & -++-+- \\
6 & 0.294 & -0.11 & -++--+ \\
7 & 0.318 & -1.16 & -+--+- \\
8 & 0.381 & -1.12 & -+-+-- \\
9 & 0.395 & -0.30 & -+-++- \\
10 & 0.464 & -0.72 & +--+-- \\
11 & 0.469 & 0.12 & +--++- \\
12 & 0.531 & 0.19 & +-++-- \\
13 & 0.604 & 1.12 & ++-+-+ \\
14 & 0.769 & -0.19 & --++-+ \\
15 & 0.787 & -1.18 & --+-+- \\
16 & 0.793 & -1.03 & --+--+ \\
17 & 0.942 & 0.16 & ++--+- \\
18 & 0.950 & 1.03 & ++-++- \\
19 & 0.996 & 0.23 & ++-+-- \\
20 & 1.188 & -1.14 & --++-- \\
21 & 1.272 & -1.06 & ---+-+ \\
22 & 1.627 & -1.99 & ---+-- \\
\hline \hline
\end{tabular}

Table 1

Collinear magnetic arrangements obtained for the $6 \mathrm{Mn} / \mathrm{Fe}(001)$ system. The signs indicate the orientation of the magnetic moments of the Mn layers with respect to the positive $\mathrm{z}$ axis (Fe bulk orientation). The first sign in each configuration refers to the surface Mn layer. $\bar{\mu}$ indicates the average magnetic moment in the $\mathrm{Mn}$ slab. Energy differences relative to the collinear ground state and average magnetic moment for the Mn layers are shown. 
ers of the slab) are obtained within a small energy range. The existence of all these metastable configurations indicates two facts: i) this system is a clear candidate to exhibit more stable noncollinear magnetic arrangements if we remove the constraint of collinearity. This is in accordance with results of Hafner and Spišák [22] for supported Mn slabs with 1 or 2 layers thicknesses; ii) This system should be particularly interesting as regard to its response to external magnetic fields. In this context, it is also interesting to note that the magnetic moments of $\mathrm{Mn}$ are quite large, being the surface and the interface moments $\left(4.0 \mu_{B}\right.$ and $3.0 \mu_{B}$, respectively) larger than the moment of the central layers (between $2.5-2.9 \mu_{B}$ ), as the effects of decreasing the number of neighbors (surface) and the influence of the effective magnetic field from the Fe layers (interface) are taken into account.

\subsection{Response to an external mag- netic field}

Starting from the system in its collinear ground state (configuration 0 in Table 1), we apply an uniform external magnetic field along the moments of Fe substrate, choosing the $z$ axis as parallel to the field. We calculate the electronic structure and magnetic configurations as functions of the field, whose intensity is increased until the system reaches the magnetic saturation. Then the field intensity is decreased to zero. In Figure 1(a) we plot the average magnetic moment in the $\mathrm{Mn}$ slab as a function of the external field. Each point in this graphic represents a self-consistent calculation. Units of the external field are referred to $B_{S}^{C}=14 \times 10^{3} \mathrm{~T}$ (index $C$ denotes "collinear" case), which is the intensity required for the system to become magnetically saturated in the collinear framework. In the magnetically saturated configuration ( $S$ in Fig. 1) all the local magnetic moments of the system are oriented along the field (also those of the Fe substrate) and reach their maximum value since the electronic structure is fully spinpolarized. It is worth to note that intermixing at the interface can decrease the spin-flip magnetic field. Calculations performed within collinear periodic Anderson model for $\mathrm{Fe} / \mathrm{Cr}$ multilayers [30] demonstrate that even the shape of hysteresis loop calculated individually for Fe and for Cr atoms changes significantly depending on the chemical structure of the interface region. In Figure 1(a) we observe both smooth and sharp changes of the average magnetization depending on the external field. When the average moment increases (or decreases) smoothly as a function of the field, the electronic structure of the system corresponds to a magnetic configuration in which the moduli of the local moments vary while preserving the relative magnetic couplings. Those regions are marked with a bidirectional arrow indicating their reversible character. When the external field reaches a critical value, it overcomes a local exchange coupling, and an irreversible change in the response is obtained. This situation is reflected by a sharp change in Figure 1(a). The system undergoes a transition to a magnetic configuration where, not only the moduli of the local moments vary, but also their 

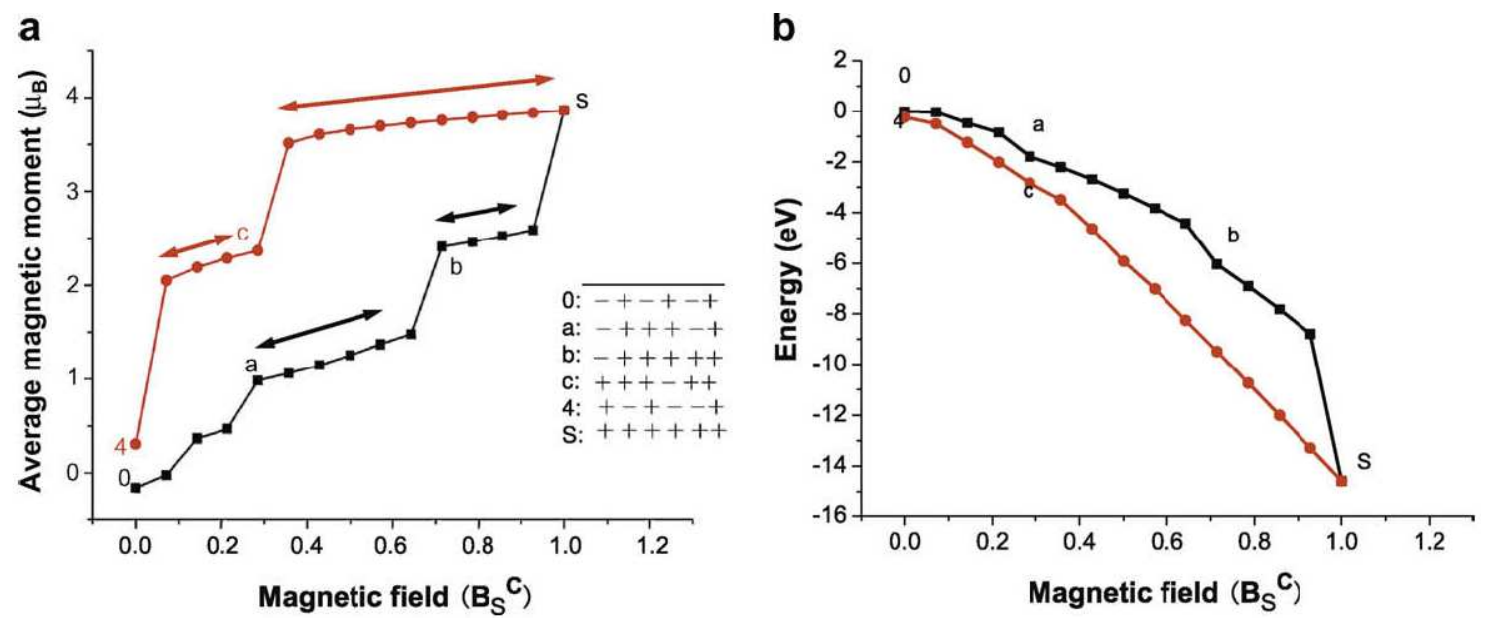

Figure 1. (a) Evolution of the average magnetic moment (collinear approach) in the Mn slab as a function of the external uniform magnetic field (in units of the saturation field $\left.B_{S}^{C}\right)$ starting from the collinear ground state: from 0 to $B_{S}^{C}$ (black squares) and from $B_{S}^{C}$ to 0 (red circles). Bidirectional arrows indicate the reversible parts of the process. (b) Evolution of the total energy in Mn slab for the process shown in Panel (a). Note that the red circle and black square at $0 T$ don't overlap, actually the vertical difference between them is the energy difference between configurations 0 and 4 in Table 1, which is about $0.2 \mathrm{eV}$.
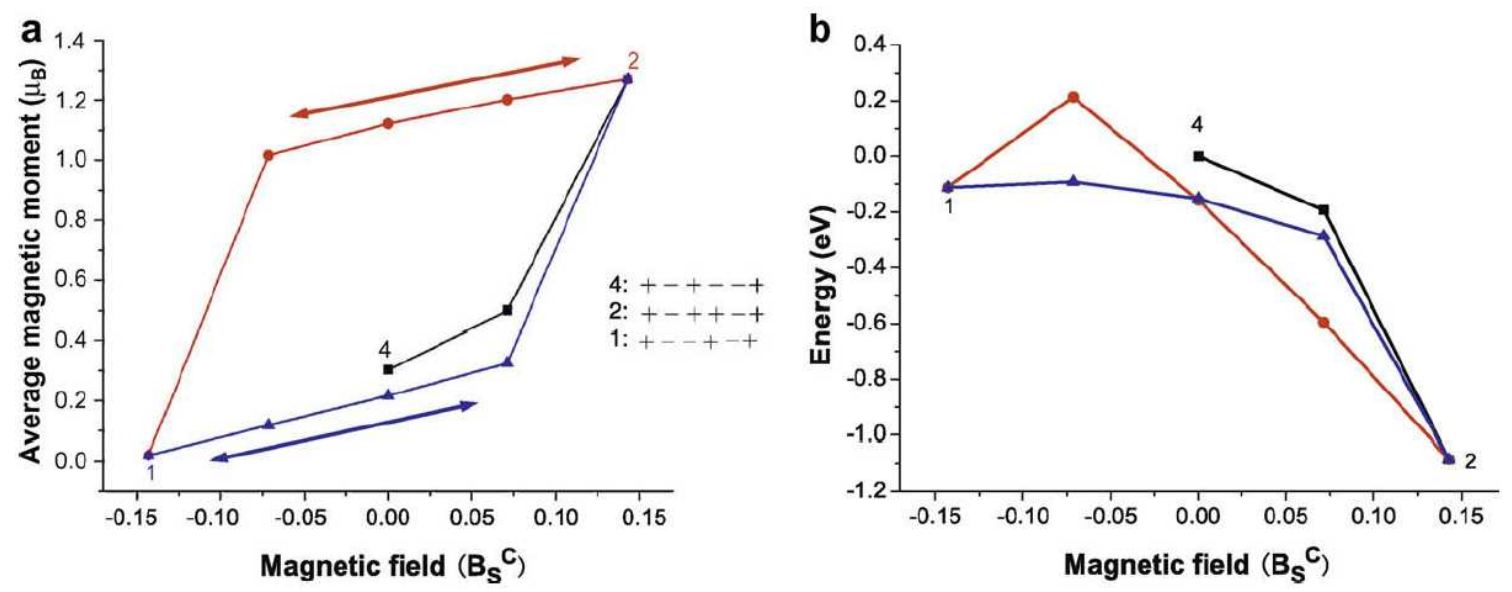

Figure 2. As in Figure 1 but starting from the metastable solution 4 and closing the cycle before reaching the magnetic saturation. 
relative exchange couplings change. If, at this point, the applied external field is reduced, the old configuration is not immediately recovered, but at an smaller applied field. The system describes then a hysteresis cycle, as can be seen in Figure 2 between solutions 2 and 1. In Figure 1(a) several of such irreversible transitions can be identified. One of them leads the system to a magnetic configuration with local exchange couplings similar to those of solution 4 in Table 1, but with different magnitudes due to the presence of the external field. There are other irreversible transitions which lead to configurations showing magnetic behaviors which are not selfconsistent solutions in the absence of external field and, therefore, are not reported in Table 1. These are, namely, $a$ $(-+++-+), b(-+++++)$, and $c$ $(+++-++)$.

By analyzing the electronic structure and the magnetic configurations of the system as increasing the external field, we come to conclusions about the relative strength of the exchange couplings at the different parts of the Mn slab. As increasing the field intensity, moments of the central layers are the first to flip and to point along the field orientation (configuration a), followed by the moments close to the interface (configuration $b$ ), and finally by the surface moments. Notice that the local moments at the surface are larger than the rest arising from the electron localization due to the lack of neighbours, that is, surface atoms are close to the atomic limit. These results indicate that the antiparallel exchange couplings at the cen- tral layers of Mn are weaker than those at the interface and than those at the surface regions, a fact reflected in the larger external fields required for demagnetization. This is consistent with the results obtained in the absence of external field, where the antiparallel couplings at the surface region and near the interface characterize the metastable solutions close to the ground state, see Table 1.

The non-reversible character of the transitions between configurations having different relative exchange couplings gives rise to the possibility of using a external magnetic field for tuning the system between different magnetic solutions which are local minima in the absence of an external field. This fact is already demonstrated in Figure 1(a). Starting from the ground state (configuration 0 ), we were able to excite the system to configuration 4 , which is a local minimum without an external field. These two solutions have similar average magnetic moment, but it is also possible to switch the system between two configurations with different average magnetization, as shown in Figure 2. In this case, starting from solution 4 , we can reach solution 2 of Table 1 well before the magnetic saturation of the system. From solution 2 we can reach solution 1, closing the cycle, by using appropriate fields. Therefore, we are able to switch the system between configuration 1 (with a low average moment of $0.22 \mu_{B}$ ) and configuration 2 (with high average magnetization of $\left.1.12 \mu_{B}\right)$, and vice versa. Although in this example we have artificially constrained the system to collinear configurations, sit- 
uations similar to the present one can be achieved in systems with a strong uniaxial anisotropy, like single molecular magnets, mentioned in the introduction.

\section{Noncollinear results}

\subsection{Intrinsic magnetic properties}

In view of the multiple magnetic solutions obtained in the collinear framework, $\mathrm{Mn}$ is a clear candidate to exhibit noncollinear effects. This possibility has been explored within the DFT approach by Hafner and Spišák[22] for one or two Mn layers supported on Fe. Larger thicknesses require a huge computational cost within DFT.

In Figure 3(a) we show the noncollinear ground state magnetic configuration obtained for six Mn layers supported on $\mathrm{Fe}(001)$. This solution presents local couplings approaching to those obtained in the ground state collinear configuration (antiparallel couplings between Mn layers, and parallel couplings at the $\mathrm{Mn} / \mathrm{Fe}$ interface and between Fe layers) but, in contrast, the surface magnetic moment is now perpendicular to the Fe bulk moment. The noncollinear ground state (Figure 3(a)) and the collinear one (solution 0 of Table 1) have otherwise similar energies, differing in few meV. The small energy difference can be understood considering that the local magnetic couplings are essentially preserved. Moreover, only one self-consistent magnetic arrangement (local minimum) is found in the noncollinear case, regardless of the input. As soon as one of the metastable configurations found in the collinear case is slightly per-

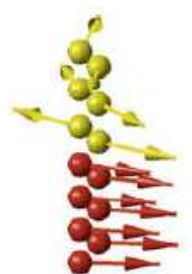

(a) 0

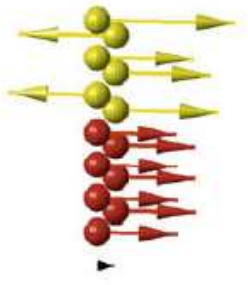

(c) $0.1 B_{S}^{N C}$

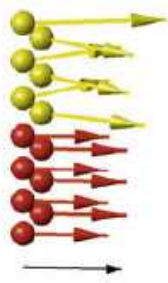

(e) $0.6 B_{S}^{N C}$

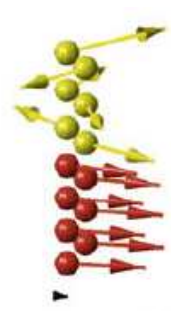

(b) $0.01 B_{S}^{N C}$

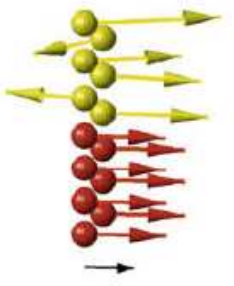

(d) $0.3 B_{S}^{N C}$

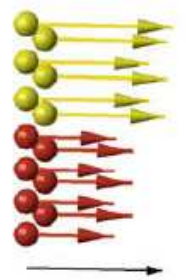

(f) $B_{S}^{N C}$
Figure 3. Selected noncollinear magnetic configurations corresponding to different values of the uniform external magnetic field applied in the $z$ direction. One atom per (001) layer is shown. The Mn atoms are represented by yellow balls while the Fe atoms are represented by red balls. The arrows are proportional to the local magnetic moments, and the direction of the magnetization in the Fe bulk is parallel to the external field which is represented by the black vector under each figure. 
turbed and introduced as input of the noncollinear calculation, it evolves towards the noncollinear ground state. The release of the collinear constraint provides a new degree of freedom to the local moments and their relative exchange couplings. We have seen that in the collinear framework the ground state can be only reached through the variations of the moduli of the local moments, and the system can get trapped in multiple local minima. Now, within the noncollinear framework, the local moments can continuously rotate during self-consistency towards the ground state. The mechanism of spin-flip is therefore different in both frameworks, and this will have important implications in the response of the system to external magnetic fields. As we will see in the next section, in the noncollinear picture the local moments will tend to rotate towards the field while preserving, at the same time, their relative exchange couplings.

\subsection{Response to an external mag- netic field}

In the noncollinear framework the local magnetic moments of the system can have both longitudinal and transversal components (referred to the direction of the moment in the bulk). In Figure 4 we plot both components of the total moment in the Mn slab as a function of the external field, starting from the noncollinear ground state shown in Figure 3(a). As in the collinear case, each point represents a self-consistent calculation. In contrast to the collinear picture, now the response is reversible because the local moments can rotate and there is only one magnetic solution for a given value of the external field. Lower applied magnetic fields are now required to change the magnetic configuration, as compared with the collinear case. For instance, the applied field needed to reach the magnetic saturation is now $B_{S}^{N C}=9.4 \times 10^{3} \mathrm{~T}$ (index $N C$ denotes "noncollinear" case), about two thirds of the value obtained in the collinear situation.

In Figure 3 we show several selected magnetic configurations corresponding to different intensities of the applied field. In absence of external field, the longitudinal component of the total magnetic moment in the Mn slab is very small $\left(1.32 \mu_{B}\right)$. As the field is applied, the total moment rotates towards the field and the longitudinal component increases rapidly (see Figure 4(a)). However, there is a competition between the tendencies of the local moments to align themselves with the field and to preserve their antiparallel exchange coupling. This is clear in the selected magnetic configurations of Figure 3 for low fields, in which not all the local moments rotate towards the field. For instance, for $B=0.01 B_{S}^{N C}$ the moment of the subsurface Mn layer tries to preserve its antiparallel coupling with the moment of the surface layer, which rotates itself towards the field. This also happens in other parts of the slab. As increasing the field up to about $0.1 B_{S}^{N C}$, the magnetic moments continue rotating; while the intensities of external magnetic fields are between this value and $0.3 B_{S}^{N C}$, the local couplings in the system are similar to those of the collinear solution 2 of Ta- 

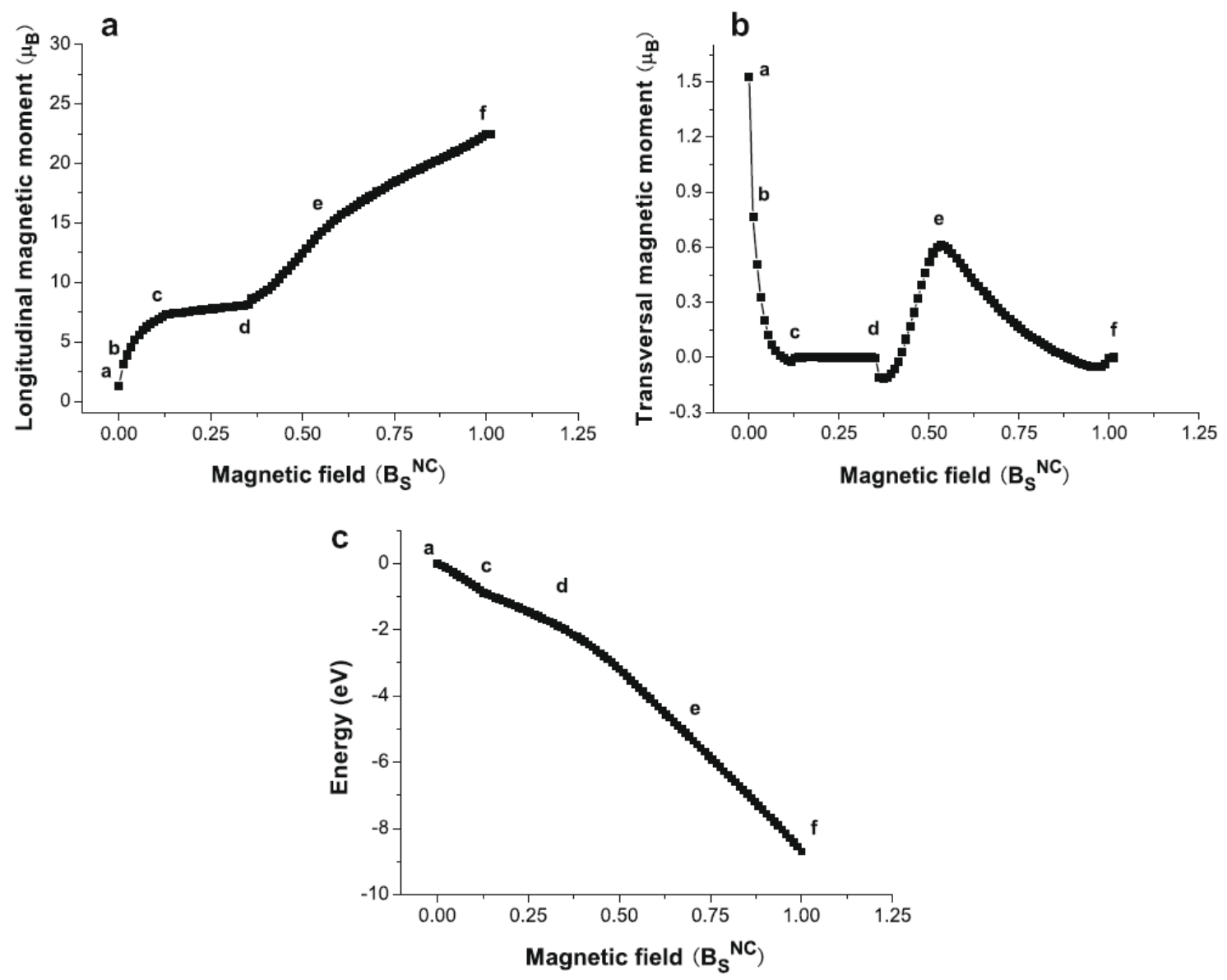

Figure 4. Evolution of the longitudinal (a) and transversal (b) components of the total magnetic moment in the Mn slab as a function of the external magnetic field (in units of the saturation field $B_{S}^{N C}$ ) starting from the noncollinear ground state. (c) Evolution of the total energy in Mn slab for the process shown in Panel (a) and (b). The symbols in the subfigures (a-f) correspond to those states presented in Figure 3. 
ble 1. In other words, the system gets trapped in a collinear magnetic configuration. This is reflected in the longitudinal and transversal components of the total magnetization in Figure 4, which stay almost constant between the referred fields. A less steep region of the energy plot in Figure 4(c) also shows this collinear configuration. For fields beyond $0.3 B_{S}^{N C}$ the moments keep rotating up to the magnetic saturation. As obtained in the collinear framework, the magnetic couplings in the central layers are weaker than those at the interface region and at the surface.

Recently experimental results by Kojima et al [31] for the $\mathrm{CdCr}_{2} \mathrm{O}_{4}$ system show a similar noncollinear remagnetisation feature at low temperature. They applied external magnetic fields up to $140 \mathrm{~T}$ in their geometrically frustrated system which saturated at $90 \mathrm{~T}$. These authors referred to a $1 / 2$ plateau phase in which $\mathrm{Cr}^{3+}$ has a 3-up 1-down collinear spin configuration. In our calculations for the $\mathrm{Mn} / \mathrm{Fe}$ system we also obtain a collinear window for field intensities between $0.1 B_{S}^{N C}$ and $0.3 B_{S}^{N C}$. As regard to the order of magnitude of the required external fields during the remagnetization process, we note that the exchange parameter $J=0.6 \mathrm{meV}$ estimated by Kojima et al for the system $\mathrm{CdCr}_{2} \mathrm{O}_{4}$ is several orders of magnitude weaker than those involved in our $\mathrm{Mn} / \mathrm{Fe}$ system $(J=1.125 \mathrm{eV}$ in manganese layers and $J=1.265 \mathrm{eV}$ in iron layers). Therefore, huge external fields are expected to be required for the remagnetization process in our case, and in particular to reach the magnetic satu- ration.

The very different behavior of the system in collinear and noncollinear frameworks emphasizes the necessity of using the second one when studying in a realistic manner systems like the one investigated here.

\section{Summary}

We have investigated the response of six Mn layers supported on $\mathrm{Fe}(001)$ to external uniform magnetic fields by solving selfconsistently a tight-binding Hamiltonian, parameterized to $a b$ initio TB-LMTO calculations, which we have extended to account for the interaction of the spinmoments with the external field. Our quantum-mechanical approach is based on electronic structure calculations and describes the remagnetization process in transition metal systems with itinerant electrons.

In the collinear framework and in absence of an external field, we have obtained multiple magnetic configurations, the ground state showing antiparallel couplings between Mn layers and parallel coupling at the $\mathrm{Mn} / \mathrm{Fe}$ interface. Similar local magnetic couplings have been obtained in the ground state configuration found in the noncollinear framework, although, in this case, the surface magnetic moment is perpendicular to the bulk moment and no other metastable configurations are obtained.

When the external field is applied, there is a competition between the tendencies of the local moments to align themselves with the external field and to preserve 
their antiparallel exchange coupling. In the collinear case with an external magnetic field only the value of the moments can vary continuously, whereas their directions can only be changed via spin flip transitions. In the noncollinear approach the local moments can both rotate and change their value continuously. The response in the collinear case shows both smooth and sharp changes of the average magnetic moment of the Mn slab depending on the external field. The smooth changes correspond to reversible variations of a magnetic configuration of the system, while the sharp ones are the result of spin flip transitions between the configurations with different local exchange couplings, and correspond to a non reversible situation. In contrast, a reversible response is always obtained in the noncollinear framework for the full range of applied fields. Therefore, the response of the system is markedly different in both approaches. Due to the non-reversibility obtained in the collinear framework, the external magnetic field can be used to switch the system between different metastable configurations, some of them having very different average magnetic moments. This situation can be achieved in real systems with strong uniaxial anisotropy.

The present study opens new prospects for investigating the response of other complex nanostructures, not only to uniform external magnetic fields, but also to non-uniform fields having different intensity and orientation in different local parts of the system (even at the atomic scale), like for example ferromagnetic or antiferromagnetic clusters supported on polarizable substrates, a situation that could be managed in the near future. Work in this line is in progress.

\section{Acknowledgments}

This work has been partially supported by the PhD program "Synthesis and Characterisation of Surfaces and Interfaces assembled from Clusters and Molecules" of the Bundesland Niedersachsen, Germany, the Spanish Ministry of Science and Innovation in conjunction with the European Regional Development fund (Project FIS2008-02490/FIS), by Junta de Castilla y León (Project GR120), and RFBR 0702-01065.

\section{REFERENCES}

1. S. D. Bader, Rev. Mod. Phys. 78, (2006) 1 .

2. P. Bruno, Phys. Rev. Lett 83, (1999) 2425.

3. C. Chappert and T. Devolder, Nature 432, (2004) 162.

4. E. Saitoh, H. Miyajima, T. Yamaoka and G. Tatara, Nature 432, (2004) 203.

5. D. Suess, Appl. Phys. Lett. 89, (2006) 113105.

6. J. Hauschild, U. Gradmann and H. J. Elmers, Appl. Phys. Lett. 72, (1998) 3211.

7. T. Eimüller, R. Kalchgruber, P. Fischer, G. Schütz, P. Guttmann, G. Schmahl, M. Köhler, K. Prügl, M. Scholz, F. Bammes and G. 
Bayreuther, J. Appl. Phys. 87, (2000) 6478.

8. V. V. Volkov and Y. Zhu, J. Magn. Magn. Matter 214, (2000) 204.

9. K. J. Kirk, J. N. Chapman, S. McVitie, P. R. Aitchison and C. D. W. Wilkinson, Appl. Phys. Lett. 75, (1999) 3683.

10. W. H. Rippard, A. C. Perrella, P. Chalsani, F. J. Albert, J. A. Katine and R. A. Buhrman, Appl. Phys. Lett. 77, (2000) 1357.

11. O. Pietzsch, A. Kubetzka, M. Bode and R. Wiesendanger, Science 292, (2001) 2053.

12. V.M. Uzdin and C. Demangeat, J. Phys.: Condens. Matter 18, (2006) 2717.

13. A. Bergman, L. Nordström, A. B. Klautau, S. Frota-Pessôa and O. Eriksson, Phys. Rev. B 73, (2006) 174434 .

14. A. Bergman, L. Norström, A. B. Klautau, S. Frota-Pessôa and O. Eriksson, Surf. Sci. 600, (2006) 4838.

15. L.Thomas, F.Lionti, R.Ballou, D. Gatteschi, R. Sessoli and B. Barbara, Nature 383, (1996) 145.

16. E.E. Fullerton, J. S. Jiang, M. Grimsditch, C. H. Sowers, and S. D. Bader, Phys. Rev. B 58, (1998) 12193.

17. A. J. Freeman and K. Nakamura, J. Magn. Magn. Mater. 321, (2009) 894.

18. S. Lounis, P. H. Dederichs and S. Blügel, Phys. Rev. Lett. 101, (2008) 107204.

19. T. K. Yamada, M. M. J. Bischoff, G. M. M. Heijnen, T. Mizoguchi and H. van Kempen, Phys. Rev. Lett. 90,
(2003) 056803.

20. U. Schlickum, N. Janke-Gilman, W. Wulfhekel and J. Kirschner, Phys. Rev. Lett. 92, (2004) 107203.

21. E. Martínez, A. Vega, R. Robles and A. L. Vázquez de Parga, Phys. Lett. A 337, (2005) 469.

22. J. Hafner and D. Spišák, Phys. Rev. B 72, (2005) 144420.

23. A. Ernst, J. Henk and R. K. Thapa, J. Phys.: Condens. Matter 17, (2005) 3269.

24. T. K. Yamada, E. Martínez, A. Vega, R. Robles, D. Stoeffler, A. L. Vázquez de Parga, T. Mizoguchi and H. van Kempen, Nanotechnology 18, (2007) 235702.

25. V.M. Uzdin and A. Vega, Phys. Rev. B 77, 134446 (2008); Nanotechnology 19, (2008) 315401.

26. R. Robles, E. Martínez, D. Stoeffler and A. Vega, Phys. Rev. B 68, (2003) 094413.

27. E. Martínez, R. Robles, D. Stoeffler and A. Vega, Phys. Rev. B 74, (2006) 184435.

28. O.K. Andersen and O. Jepsen, Phys. Rev. Lett. 53, (1984) 2571.

29. R. Haydock, in Solid State Physics, edited by H. Ehrenreich, F. Seitz, and D. Turnbull (Academic, New York, 1980), Vol. 35, p. 215.

30. V.M. Uzdin and N.S. Yartseva, J. Magn. Magn. Mat. 203, (1999) 280.

31. E. Kojima, A. Miyata, et al, Phys. Rev. B 77, (2008) 212408. 